\title{
Molecular Dynamics Study of Carbon Diffusion in Austenite
}

\author{
A.V. Evteev ${ }^{1, a}$, E.V. Levchenko ${ }^{1, b}$, I.V. Belova ${ }^{1, c}$ and G.E. Murch ${ }^{1, d}$ \\ ${ }^{1}$ Diffusion in Solids Group, School of Engineering, \\ The University of Newcastle, Callaghan, NSW 2308, Australia \\ aAlexander.Evteev@newcastle.edu.au, ${ }^{\mathrm{b}}$ Elena.Levchenko@newcastle.edu.au, \\ Irina.Belova@newcastle.edu.au, 'Graeme.Murch@newcastle.edu.au
}

Keywords: Atomistic modelling, molecular dynamics, interstitial diffusion, austenite

\begin{abstract}
The mmolecular dynamics method is applied to investigate carbon interstitial diffusion in austenite at low carbon content. An approximation that carbon atoms can interact with each other only indirectly (via neighbouring iron atoms) is used. Sets of Arrhenius parameters of interstitial carbon jump frequencies identified by the four-frequency model are determined. Comparison of the molecular dynamics results with experimental data analysis in the context of the four-frequency model is performed. It is shown that the four-frequency model may not be adequate to describe the carbon diffusion process. To improve the analytical model the specific role of the transition probabilities during association and dissociation of the first nearest neighbour carbon pairs through the second neighbour sites should be considered. The direct repulsion between the carbon first neighbour positions should be also taken into account in molecular dynamics simulation.
\end{abstract}

\section{Introduction}

Carbon interstitial diffusion in austenite $(\gamma-\mathrm{Fe})$ has been studied by experimental and analytical methods for many years. Two experimental studies have been made of carbon diffusion in austenite. One is a measurement of the chemical or intrinsic diffusion coefficient at $1075 \mathrm{~K}, 1124 \mathrm{~K}$ and $1273 \mathrm{~K}$, and as a function of carbon composition [1]; the other is a measurement of the tracer carbon diffusion coefficient at $1273 \mathrm{~K}$ as a function of carbon composition [2]. These data have been analysed on many occasions with two rather different diffusion kinetics models [2-7].

The first model is the well-known interacting lattice gas model in which effective nearest neighbour pair interactions are supposed between the interstitial atoms and an inter-site transition probability based on those interactions is formulated; see, for example, Kikuchi and Sato [8]. Repulsive interactions between the carbon atoms are required in order to make quantitative contact with the carbon diffusion data. A difficulty with the lattice gas model is one of uniquely describing 'rotational jumps' (a second atom making a rotational jump from one nearest neighbour site to another of a given atom) in the f.c.c. lattice when simple inter-site transition probabilities are used.

In the second model, which is appropriate only at very low carbon content, it is supposed that the diffusing species can diffuse only as isolated atoms or as bound (nearest neighbour) pairs. Because this model is based on a specification of fundamental jump frequencies it is a rather more general model than one starting from effective interactions from which jump frequencies can be derived. In this second model, four atom - vacant site exchange frequencies are explicitly specified: $w_{0}$ is the atom - vacant site exchange frequency for jumps of isolated interstitial atoms; $w_{1}$ is a rotational atom - vacant site frequency of one atom about the other when the pair interstitial atoms are first nearest neighbours; $w_{3}$ is the dissociative exchange frequency between one of atoms forming a first nearest neighbour interstitial pair and the second, third or fourth neighbour vacant sites with respect to another atom of the first nearest neighbour interstitial pair; $w_{4}$ is the frequency of the reverse (associative) jumps to form a first nearest neighbour interstitial pair. All other interstitial jumps are assumed in this model occur to have the same frequency $w_{0}$ as for an isolated interstitial atoms. By making use of the exact solution to the four-frequency model [9] the ratios of the interstitial jump frequencies $w_{0} / w_{3}$ and $w_{1} / w_{3}$ were calculated [10]. Taking into account results of the previous study [7] the resulting limits for these ratios at $1273 \mathrm{~K}$ are rather wide: $0.7<w_{1} / w_{3}$ $<6.6 ; 0.6<w_{0} / w_{3}<1.5$. Experimental thermodynamic data $[11,12]$ can be easily connected to $w_{4} / w_{3}-$ ratio of the associative to the dissociative jump frequencies. Then $w_{4} / w_{3}$ lies between 0.45 and 0.6 for the temperatures $1173-1373 \mathrm{~K}$. This means that there is a moderately repulsive (effective) interaction between interstitial carbon atoms. 
In the present paper, we use molecular dynamics (MD) simulation to test these findings. MD simulation can provide information on all of the four types of interstitial atom jump frequencies.

\section{The Model}

For the calculation of the jump frequencies of the isolated interstitial carbon atoms we have used the model referred to as Model 1; for the calculation of the rotational and dissociate jump frequencies of carbon interstitial pairs we used the model referred to as Model 2; and for the calculation of the associative jump frequency of carbon interstitial pairs we used the model referred to as Model 3.

The austenite structure was simulated as a calculation cube with periodic boundary conditions consisting of $24 \times 24 \times 24$ f.c.c. unit cells and containing 55296 iron atoms. The lattice parameter of austenite as a function of the carbon concentration $c$ (fraction of occupied interstitials sites) was calculated according to an empirical expression derived on the basis of experimental data [13]: $a=3.572+0.78 c \AA$.

As an initial state of Model 1 the carbon atoms (a total of 256 atoms) were placed in the octahedral voids of the austenite lattice in a regular way $(4 \times 4 \times 4$ f.c.c. unit cells) and the distance between the nearest carbon atoms was $15.17 \AA$ or at least 6 elementary jump distances. For the purposes of the MD simulation this type of separation was considered to be sufficient to exclude any 'measurable' influence of the other interstitial atoms. For the initial state of Model 2 an extra 256 carbon atoms were added to the carbon atoms of Model 1 and placed in the first nearest octahedral void to form isolated 256 pairs. For the initial state of Model 3 an extra 256 carbon atoms were added to the carbon atoms of Model 1 and placed in the second nearest octahedral void positions. The effective concentration of carbon atoms was $\sim 0.46$ at. $\%$ in Model 1 and $\sim 0.92$ at. \% in Models 2 and 3. To ensure that the separation of atoms and pairs of atoms was sufficient in the MD simulation a test calculation was performed for Model 2 with the composition of carbon atoms $\sim 4.6$ times lower than the chosen one. Both models give the same results.

The Fe-Fe interaction was described by the well-known Johnson empirical pair-potential [14]:

$$
\varphi_{\mathrm{Fe}-\mathrm{Fe}}(r)= \begin{cases}-2.195976(r-3.097910)^{3}+2.704060 r-7.436448 \mathrm{eV}, & r<2.40 \AA, \\ -0.639230(r-3.115829)^{3}+0.477871 r-1.581570 \mathrm{eV}, & 2.40 \AA \leq r<3.00 \AA, \\ -1.115035(r-3.066403)^{3}+0.466892 r-1.547967 \mathrm{eV}, & 3.00 \AA \leq r \leq 3.44 \AA .\end{cases}
$$

The Johnson potential and its first derivative smoothly go to zero at a cut-off radius of $3.44 \AA$. The parameters for this potential have been chosen in such a way that the elastic and dynamic properties of the $\alpha$-Fe were matched. This potential represents well the behaviour of the Fe interstitials, vacancies and their complexes in $\alpha$-iron $[14,15]$. Later, Johnson showed [16] that the same potential can be also used for $\gamma$-Fe and it allows for the adequate description of the elastic and dynamic properties as well as the behaviour of the point defects and their complexes. A discussion and proof of the validity of such a seemingly arbitrary choice for the f.c.c. interaction is presented in [16].

To describe the Fe-C pair interaction we used the potential proposed by Johnson et al. [17]:

$$
\varphi_{\mathrm{Fe}-\mathrm{C}}(r)=-\varepsilon\left[2\left(\frac{r-r_{\mathrm{c}}}{r_{\mathrm{c}}-r_{0}}\right)^{3}+3\left(\frac{r-r_{\mathrm{c}}}{r_{\mathrm{c}}-r_{0}}\right)^{2}\right] \text {. }
$$

Fitting of the Johnson-Dienes-Damask potential to the experimental data for $\alpha$-Fe (the migration energy of carbon atoms, the activation volume of carbon migration and the vacancy-carbon binding energy) was done using for the Fe-Fe Johnson potential (Eq. 1) in a set of computer experiments. Three critical parameters have been changed: the position $r_{0}$, the depth $\varepsilon$ of the potential well and the potential cut-off radius $r_{\mathrm{c}}$. The final values for these parameters are: $r_{0}=1.94 \AA, \varepsilon=0.35 \mathrm{eV}, r_{\mathrm{c}}=2.53 \AA$ [17]. The use of the Johnson-Dienes-Damask potential together with the Johnson potential allows for the adequate description of the behaviour of carbon interstitials in the $\alpha$-Fe [17-19] and in martensite [20]. The bodycentred tetragonal phase of iron, and the structure and energy of cementite [17] were also investigated by means of these potentials. The use of the Johnson-Dienes-Damask potential together with the empirical Pak-Doyama pair potential [21] (this is very similar to the Johnson potential) provides good agreement between the structural characteristics of the MD model of the Fe-C melts and experimental data [22]. At 
the same time, this combination of potentials provides an explanation of the low glass forming ability of the metallic alloys of this system compare to the metallic Fe-B and Fe-P alloys [22]. More recently, the Johnson-Dienes-Damask potential has been used in a series of studies of the carbon nano-tube growth on the surface of the iron cluster; see, for example, [23].

The situation with the carbon-carbon potential is more complicated. There is no agreed and detailed data about the preferred type of interaction for carbon-carbon pairs in a dilute iron-carbon solid solution. However, it is clear that this interaction is very weak and that is why as a first approximation it can be considered as a small perturbation to the much stronger $\mathrm{Fe}-\mathrm{Fe}$ and $\mathrm{Fe}-\mathrm{C}$ interactions. In the present study a purely repulsive Born-Mayer potential [24]:

$$
\varphi_{\mathrm{C}-\mathrm{C}}(r)=A \exp \left(-r / r_{\mathrm{s}}\right)
$$

(with the energy parameter $A=764.2 \mathrm{eV}$ and the length of screening $r_{\mathrm{s}}=0.219 \AA$ proposed in [25] and a cut-off radius of $2 \AA$ ) was selected for describing the C-C interaction. This potential is used because the only task here is to prevent the situation when two carbon atoms occupy the same octahedral void.

To initiate the diffusion process, atoms were given initial velocities according to the usual Maxwell distribution at a given temperature, and isothermal annealing procedures were performed in a temperature range of 1173-1523 K (for Model 1) and 1373-1523 K (for Models 2 and 3) with a step of $50 \mathrm{~K}$. The MD simulations consist of a numerical integration of the equations of atomic motion using a time step $\Delta t=1.5 \times 10^{-15} \mathrm{~s}$ according to the well-known Verlet algorithm [26]. The duration of every MD annealing cycle at any temperature was $40000 \Delta t$ or $60 \mathrm{ps}$. The total numbers of MD annealing cycles at any temperature were $12(0.72 \mathrm{~ns}), 27$ (1.62 ns) and 20 (1.2 ns) for Models 1, 2 and 3 respectively. After every cycle, the system was transferred to a state at $T=0 \mathrm{~K}$ where atoms occupied equilibrium positions in local potential minima. This was done by making use of the static relaxation method. After this, the carbon movements occurring in the current model were analyzed.

In the present work two methods have been performed to analyze the MD diffusion data. One is based on the calculation of atomic mean square displacements (MSD) in a MD model (MSD method) and other is derived from the direct counting of the jumps observed in a MD model (jumps method). We used the MSD method just for Model 1, since only in this case are all the jumps of the same kind (jumps of isolated carbon atoms) and have the same average frequency. Therefore, only for Model 1 it is reasonable to compare activation parameters obtained by both MSD and jumps methods.

\section{Results and Discussion}

Model 1. The (tracer) diffusion coefficient $D$ at each temperature was calculated using the Einstein equation $\left\langle\Delta r^{2}(t)\right\rangle=6 D t$ from the MSD of the carbon atoms (Fig. 1). The (apparent) diffusion coefficient of isolated interstitial carbon atoms can be expressed in terms of the jump frequency $w_{0}$ as:

$$
D=\frac{1}{6} z b^{2} f w_{0}=\frac{1}{6} z b^{2} f v_{0} \exp \left(\Delta S_{0} / R\right) \exp \left(-E_{0} / R T\right)=D_{0} \exp \left(-E_{0} / R T\right),
$$

where $z$ is the coordination number, $b$ is the average jump distance, $f$ is the tracer correlation factor, $v_{0}$ is the effective attempt frequency, $E_{0}$ and $\Delta S_{0}$ are the activation energy and entropy of a jump of an isolated interstitial carbon atom, $R$ is the ideal gas constant. The temperature dependence of the diffusion coefficient $D$ obtained from MSD is shown in Fig. 2 as filled circles. Using the Arrhenius-type equation Eq. 4 (the solid line in Fig.2), the activation parameters (activation energy $E_{0}$ and preexponential factor $D_{0}$ ) of diffusion of isolated interstitial carbon atoms were calculated (Table 1). We also found a value of $v_{0} \exp \left(\Delta S_{0} / R\right)$ multiplier (Table 1) within the framework of the MSD method from the pre-exponential factor $D_{0}$ by taking $z=12, b=2.5283 \AA$ and $f=1$.

On the other hand, the jump frequency of isolated interstitial carbon atoms can be written as:

$$
w_{0}=n_{0} / 12 N_{\mathrm{C}} t \text {, }
$$

where $n_{0}$ is the average number of the jumps of isolated carbon atoms occurring in MD model during time $t$ and $N_{\mathrm{C}}=256$ is the number of carbon atoms in the model. As an example, the 
frequency $w_{0}$ was calculated by averaging over 516 jumps and over 20 jumps at the highest temperature $(1523 \mathrm{~K})$ and at the lowest temperature $(1173 \mathrm{~K})$ from investigated range respectively. The Arrhenius plot of $\ln \left(w_{0}\right)$ vs $T^{1}$ calculated by means of Eq. 5 is shown in Fig. 2 (stars). The activation energy $E_{0}$ and the multiplier $v_{0} \exp \left(\Delta S_{0} / R\right)$ obtained by the fitting (the dash line in Fig. 2) of these data and calculated $D_{0}$ are also presented in Table 1.

We can see from Fig. 2 that the diffusion data obtained by means of the two different methods of analysis are very close to each other. This confirms that diffusion of isolated interstitial carbon atoms in austenite proceeds by interstitial jump mechanisms with a tracer correlation factor $f=1$.

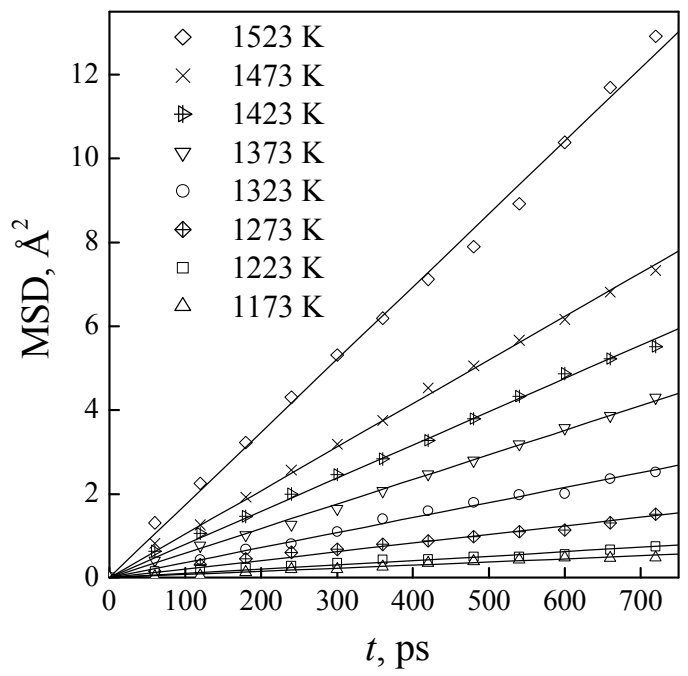

Figure 1. Time dependences of the MSD of the carbon atoms in Model 1 at various temperatures. Solid lines represent approximation by the Einstein equation for a given temperature.

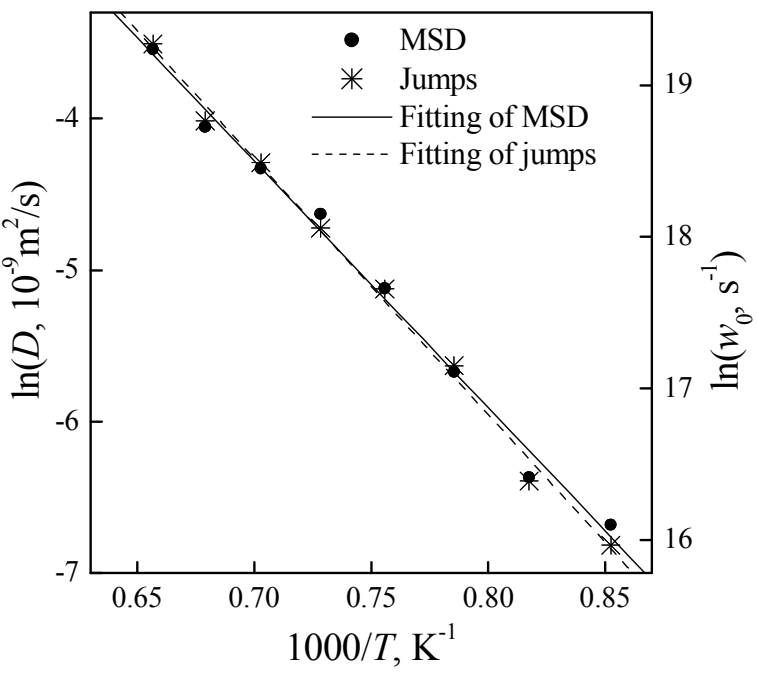

Figure 2. Comparison of results from MSD and jumps methods for Model 1 and test of the Arrhenius behaviour of the diffusion coefficient $D$ (RH scale) and the jump frequency (LH scale) of isolated carbon atoms.

Table 1. Comparison of calculated and experimental Arrhenius parameters of carbon interstitial diffusion in austenite

\begin{tabular}{|c|c|c|c|c|c|c|}
\hline \multirow{3}{*}{$\begin{array}{l}\text { Activation } \\
\text { parameters }\end{array}$} & \multicolumn{5}{|c|}{ Calculated in this work } & \multirow{3}{*}{$\begin{array}{l}\text { Experimental } \\
\text { data }^{2} \text { [27-29] }\end{array}$} \\
\hline & \multirow[b]{2}{*}{ Method } & Model 1 & \multicolumn{2}{|c|}{ Model 2} & \multirow{2}{*}{$\begin{array}{c}\text { Model } 3 \\
\text { Association } \\
\text { of pairs }\end{array}$} & \\
\hline & & $\begin{array}{l}\text { Isolated } \\
\text { atoms }\end{array}$ & $\begin{array}{l}\text { Rotation } \\
\text { of pairs }\end{array}$ & $\begin{array}{l}\text { Dissociation } \\
\text { of pairs }\end{array}$ & & \\
\hline \multirow{2}{*}{$E[\mathrm{~kJ} / \mathrm{mol}]$} & MSD & 135.2 & - & - & - & \multirow{2}{*}{$\begin{array}{c}154.1 ; 156.84 \\
147.81\end{array}$} \\
\hline & Jumps & 140.3 & 181.7 & 144.8 & 143.4 & \\
\hline \multirow{2}{*}{$D_{0}\left[10^{-5} \mathrm{~m}^{2} / \mathrm{s}\right]$} & MSD & 0.12 & \multicolumn{2}{|r|}{-} & - & \multirow{2}{*}{$4.5 ; 6.68 ; 2.34$} \\
\hline & Jumps & 0.19 & & - & - & \\
\hline \multirow{2}{*}{$\begin{array}{c}v_{0} \exp (\Delta S / R) \\
{[\mathrm{THz}]}\end{array}$} & MSD & 9.5 & - & - & - & \\
\hline & Jumps & 14.9 & 404.6 & 17.0 & 19.6 & \\
\hline
\end{tabular}

${ }^{1}$ Only from the sites of third neighbour shell;

${ }^{2}$ Corresponds to all jumps without separation on isolated, rotational, dissociative or associative jumps.

Model 2. We used this model to determine the rotational and dissociative carbon jumps frequencies. Taking into account that rotation and dissociative jumps have 8 and 14 possible directions per pair respectively, we find that:

$$
w_{1}=n_{1} / 8 n_{\mathrm{CC}}^{(1)} t, \quad w_{3}=n_{3} / 14 n_{\mathrm{CC}}^{(1)} t,
$$


where $n_{1} / n_{\mathrm{CC}}^{(1)}$ and $n_{3} / n_{\mathrm{CC}}^{(1)}$ are the average fractions of the rotation and the dissociation jumps respectively per one pair from any number of $n_{\mathrm{CC}}^{(1)}$ current (at initial state $n_{\mathrm{CC}}^{(1)}=256$ ) carbon-carbon first nearest neighbour pairs in MD model during time $t$. In the investigated temperature range the total numbers of carbon jumps were 2052 and 513 at the highest $(1523 \mathrm{~K})$ and the lowest $(1373 \mathrm{~K})$ temperatures respectively. The Arrhenius parameters obtained by fitting to the calculated rotation and dissociation jumps frequencies (Fig. 3) are listed in Table 1.

Model 3. During the analysis the data of this model it was found that in contrast with dissociation, the rate of association significantly depends on the site from which it occurs. Given the initial state of this model it should be pointed out that the majority of the associating atoms firstly jumped from the second neighbour sites to the third neighbour sites and only after this formed first nearest neighbour pairs. Therefore it is impossible for this model to determine satisfactorily the temperature dependence of the association rate from the second neighbour sites. Although it was established for the whole investigated temperature range that the association rate from the second neighbour sites is $8-9$ times lower than from the third neighbour sites. It can be reasonably explained from a physical point of view that the iron atom located directly between two second neighbour carbon atoms effectively increases their repulsive interaction. And of course this model does not allow us to determine the association rate from fourth neighbour sites. However, we can suppose that it will not differ greatly from the association rate from the third neighbour sites.

We define $w_{4}{ }^{\prime}, w_{4}{ }^{\prime \prime}$ and $w_{4}{ }^{\prime \prime \prime}$ as associative frequencies of interstitial carbon atoms from the second, third and fourth neighbour sites respectively. Then, considering that we have 6 second, 24 third and 12 fourth neighbour sites with 4, 2 and 1 possible associative jumps per site respectively, the average associate jump frequency can be roughly estimated as:

$$
w_{4}=\left(2 w_{4}{ }^{\prime}+4 w_{4}{ }^{\prime \prime}+w_{4}{ }^{\prime \prime}\right) / 7 \approx 5 w_{4}{ }^{\prime \prime} / 7
$$

and calculated from this model (associative jump from third neighbour sites has 4 possible directions per pair):

$$
w_{4}{ }^{\prime \prime}=n_{4}{ }^{\prime \prime} / 4 n_{\mathrm{CC}}^{(3)} t,
$$

where $n_{4}{ }^{\prime \prime} / n_{\mathrm{CC}}^{(3)}$ is the average fraction of the association jumps from the third neighbour sites per one pair from any number of $n_{\mathrm{CC}}^{(3)}$ current carbon-carbon third neighbour pairs in the MD model during time $t$. For

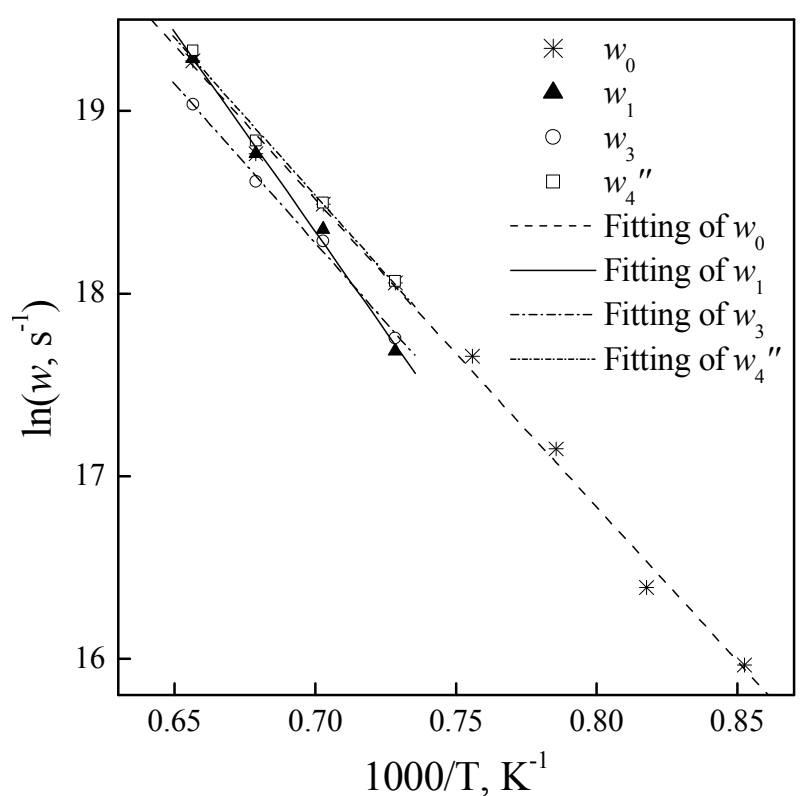

Figure 3. The four jump frequencies for carbon interstitial diffusion in dilute austenite according to the molecular dynamic simulation. instance, during the entire time of the MD experiment 1520 carbon jumps were observed at $1523 \mathrm{~K}$ and 420 carbon jumps were at $1373 \mathrm{~K}$. The activation parameters obtained by Arrhenius fitting of the calculated frequency of associative jumps from the third neighbour sites (Fig. 3) are shown in Table 1.

Thus, the obtained MD results allow to express $w_{1}, w_{3}$ and $w_{4}$ in term of $w_{0}$ at $1273 \mathrm{~K}$ and then to compare with the analysis [10] of experimental data $[1,2,11,12]$ within framework of four-frequency model [7,9]. At $1273 \mathrm{~K}$ we have $w_{1} \approx 0.54 w_{0}$, $w_{3} \approx 0.75 w_{0}$ and $w_{4} \approx 0.7 w_{0}\left(w_{4}^{\prime \prime} \approx 0.98 w_{0}\right)$. Although the ratios of the interstitial jump frequencies $w_{0} / w_{3} \approx 1.33$ and $w_{1} / w_{3} \approx 0.72$ are inside the range defined in [10], but the ratio $w_{4} / w_{3} \approx 0.93$ disagrees with this analysis. If we consider that the four-frequency model adequately describes the behaviour of carbon atoms in austenite, then the results of the present MD simulation indicate that direct repulsive interaction between first nearest neighbour carbon atoms should be taken into 
account. In any case, the present MD simulations evidently demonstrate a special role of second neighbour carbon sites for association and dissociation. For example, we can expect due to the increase of the mentioned above effective repulsion between carbon atoms through a common iron atom, that a dissociative jump of an atom of the first nearest neighbour site to the second neighbour site will very soon lead to the correlated jump of any atom of the pair either to the third or the fifth neighbour sites with respect to the other one. Therefore, the effective dissociative rate through the second neighbour sites will be higher, than via third or fourth neighbour sites and as a result, the effective dissociative rate in average will increase. Application of the Johnson potential for Fe-Fe pairs and the Johnson-Dienes-Damask potential for Fe-C pairs confirms by good agreement between experimental [27-29] and the calculated activation energies for carbon diffusion in austenite (Table 1).

\section{Summary}

We have applied molecular dynamics simulation to investigate carbon diffusion in austenite at low carbon contents. Comparison of the molecular dynamics results with experimental data analysis in the context of the four-frequency model has been made. It was shown that the four-frequency analytical model may not be adequate for describing the carbon diffusion process. To improve the analytical model the specific role of the transition probabilities during association and dissociation of the first nearest neighbour carbon pairs through the second neighbour sites should be considered. The direct repulsion between the carbon first neighbour positions should be also taken into account in further molecular dynamic simulation.

\section{Acknowledgements}

We wish to thank the Australian Research Council for its support of this research.

\section{References}

[1] R.P. Smith: Acta Metall. Vol. 1 (1953), p. 578

[2] D.C. Parris and R.B. McLellan: Acta Metall. Vol. 24 (1976), p. 523

[3] R.B. McLellan: J. Phys. Chem. Solids Vol. 38 (1977), p. 933

[4] R.H. Siller and R.B. McLellan: Trans TMS-AIME, Vol. 245 (1969), p. 697

[5] R.H. Siller and R.B. McLellan: Metall. Trans. Vol. 1 (1970), p. 985

[6] G.E. Murch and R.J. Thorn: J. Phys. Chem. Solids Vol. 40 (1979), p. 389

[7] R.A. McKee: Phys. Rev. B Vol. 22 (1980), p. 2649

[8] R. Kikuchi and H. Sato: J. Chem. Phys. Vol. 55 (1971), p. 702

[9] Y. Okamura and A.R. Allnatt: Phil. Mag. A Vol. 48 (1983), p. 387

[10] I.V. Belova and G.E. Murch: Phil. Mag. Vol. 85 (2005), p. 4515

[11] S. Ban-ya, J.F. Elliott and J. Chipman: J. Metall. Trans. Vol. 1 (1970), p. 1313

[12] H. Aaronson, H.A. Domian and G.M. Pound: Trans TMS-AIME Vol. 236 (1966), p. 753

[13] K.F. Laner, J. Desimoni and G.J. Zarragoicoechea: Hyperfine Interactions Vol. 134 (2001), p. 171

[14] R.A. Johnson: Phys. Rev. Vol. 134 (1964), p. A1329

[15] J.R. Beeler, Jr. and R.A. Johnson: Phys. Rev. Vol. 156 (1967), p. 677

[16] R.A. Johnson: Phys. Rev. Vol. 145 (1966), p. 423

[17] R.A. Johnson, G.J. Dienes and A.C. Damask: Acta Metall. Vol. 12 (1964), p. 1215

[18] R.A. Johnson, A.C. Damask: Acta Metall. Vol. 12 (1964), p. 443

[19] R.A. Johnson: Acta Metall. Vol. 15 (1967), p. 513

[20] R.A. Johnson: Acta Metall. Vol. 13 (1965), p. 1259

[21] H.M. Pak, M. Doyama: J. Fac.Eng. Univ. Tokio B Vol. 30 (1969), p. 111

[22] A.V. Evteev, A.T. Kosilov, and E.V. Levtchenko: Acta Mater. Vol. 51 (2003), p. 2665

[23] F. Ding, A. Rosen and K. Bolton: J. Chem. Phys. Vol. 121 (2004), p. 2775

[24] W. Eckstein: Computer Simulation of Ion-Solid Interactions (Springer-Verlag, Berlin 1991).

[25] H.H. Andersen and P. Sigmund: J. Nucl. Instr. Meth. Vol. 38 (1965), p. 238

[26] L. Verlet: Phys. Rev. Vol. 159 (1967), p. 98

[27] C. Wells, W. Batz and R.F. Mehl: Trans. Metall. Soc. AIME Vol. 188 (1950), p. 1174

[28] R.P. Smith: Trans. Metall. Soc. AIME Vol. 230 (1964), p. 476

[29] J. Ågren: Scr. Metall. Vol. 20 (1986), p. 1507 